\title{
Update on the APOKASC analysis of Red Giants
}

\author{
Yvonne Elsworth ${ }^{1,2, \star}$ \\ ${ }^{1}$ School of Physics and Astronomy, University of Birmingham, Birmingham B15 2TT, UK \\ ${ }^{2}$ Stellar Astrophysics Centre, Department of Physics and Astronomy, Aarhus University, Ny Munkegade 120, DK-8000 Aarhus C, \\ Denmark
}

\begin{abstract}
APOKASC is a collaboration between APOGEE's spectroscopic survey in the near IR at Apache Point (SDSS) and Kepler Asteroseismic Science Consortium (KASC) for the study of red-giant stars. The aim of the collaboration is to take advantage of the good spectroscopic data of APOGEE together with the excellent KASC asteroseismic data from the Kepler mission to derive reliable and precise masses and ages for a large number of red-giant stars by combining data from the two sources. We report here on the progress to date on the just over 6600 stars being considered.
\end{abstract}

\section{Introduction}

The APOKASC consortium was formed to take advantage of the good spectroscopic data of APOGEE and the excellent asteroseismic data from the Kepler mission to derive reliable and precise masses and ages for a large number of red-giant stars. The first results on 1916 red-giant stars were published in 2014[1]. Since then, the number of stars observed has been considerably expanded, longer duration Kepler datasets are available and the spectroscopic pipeline of APOGEE has been improved. We report here on the progress with the grid-based modelling used to derive stellar masses and other parameters for just over 6600 red-giant stars. We take advantage of the latest advances in stellar modelling for stars on the upper portion of the red-giant branch where homologous scaling breaks down. Additionally, we are able to use the full precision of the asteroseismic data because data from several pipelines are provided as inputs to the grid-based modelling and it is now possible to provide information on the evolutionary state of individual stars based on the asteroseismic information and the effective temperature of the star.

\section{The Asteroseismic Data}

The average asteroseismic parameters of $v_{\max }$ and $\Delta v$ have been provided by five different teams. The methods used by the teams are described in A2Z[2], CAN[3], COR[4], OCT[5], and SYD[6]. The Kepler data used in the analysis was prepared by various different preparation methods[7] [8][Mathur et al, in preparation] and it was checked that the preparation method had no significant impact on the derived parameters. From the asteroseismic spectra, it is possible to estimate the evolutionary state of the stars, i.e. has Helium started to be burnt in the core of the star? There

\footnotetext{
^e-mail: y.p.elsworth@bham.ac.uk
}

are diverse ways of determining the evolutionary state and the detail of the methods used and the method to combine the different categorisation is given in Elsworth et al. (in preparation). In essence, the combination process looks for majority agreement between the different categorisation methods. For a summary of the different methods used see Elsworth et al.(submitted). In only about $8 \%$ of the stars is there not a reasonable consensus. These parameters, together with spectroscopic parameters from APOGEE DR13[9] are used as inputs to the grid-based modelling in order to determine the mass and other properties of the individual stars. The normal practice is to choose one preferred set of average asteroseismic parameters and to then inflate the uncertainties of that method to reflect the range of values provided by the other methods. We have chosen not to follow that route. Instead we have supplied all the data (after a small amount of extreme outlier rejection) to the grid-based modelling together with their original uncertainties. We do this to take full advantage of the precision in the average asteroseismic parameters and to explore the systematic effects of the different methods.

The asteroseismic $v_{\max }$ values have an additional use in that they are believed to provide precise and accurate estimates of the surface gravity of the stars.

\section{Goals of the Project}

From the average asteroseismic parameters and the effective temperature, it is possible to use the principles of homology to compute mass and radius. For this we use the following scaling relations[10] which can be re-arranged to provide standard expressions for mass and radius. The constant of proportionality is provided by the solar reference values for $\Delta v, v_{\max }$ and $T_{\text {eff }}$ which are specific to the individual methods. 

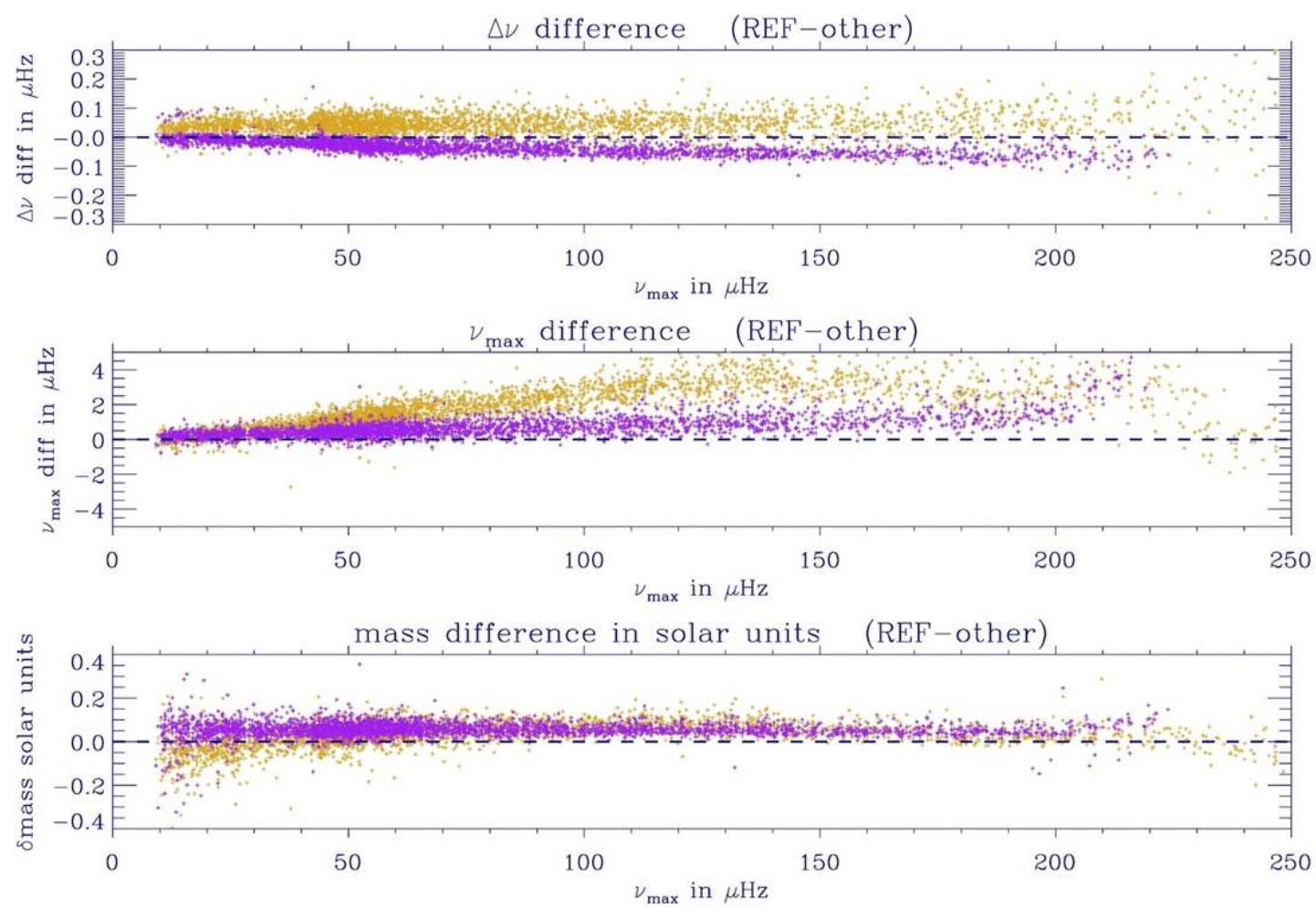

Figure 1: The average seismic parameters and the computed scaling-law mass are compared for 3 different methods (CAN, COR and OCT). In all cases the reference result is provided by CAN. In purple is CAN-COR and in light brown is CAN-OCT.

$$
\begin{gathered}
\Delta v \propto \sqrt{\frac{M}{R^{3}}} \\
v_{\max } \propto \frac{M}{R^{2} \sqrt{T_{\text {eff }}}}
\end{gathered}
$$

It is well-known that the different methods of measuring the average seismic parameters give slightly different values for the mass and the radius of stars. In other words, the solar reference values do not always fully compensate for the different approaches. Consider the data shown in figure 1. Here data from three methods are compared. The data from the CAN method are taken as the reference point. In the upper panel are shown, in light brown, the differences between $\Delta v$ from CAN and OCT as a function of the CAN $v_{\max }$ value. The sense of the comparison is CAN-OCT. In green are the differences for CAN-COR. As is evident from this plot, the $\Delta v$ values for the different methods do not precisely agree. The level of the disagreement is up to $10 \%$. This is well known and has been extensively studied (see e.g.[11]). The second panel shows the comparison for $v_{\max }$ and the final one shows the scalinglaw mass. The solar reference values have been used to mitigate the effects of the different seismic methods and it can be seen the OCT and COR now agree quite well but there are still small differences from the CAN result. Similar conclusions can be demonstrated for the other methods. The position is actually more complicated than shown here because the differences between the derived masses are actually somewhat dependent on the evolutionary states of the stars concerned.

We hope that the use of grid-based modelling will remove this disagreement. Hence, one of the major aims of the project is to measure the improvement that is gained for the giants from using grid-based modelling as opposed to the simple scaling laws. The ages of the stars, a very important parameter, can only be gained from the models. Furthermore, to give validity to the computed ages, we need to be sure that the mass calculation is precise and accurate and it is for that reason that we use both the simple scaling laws and the grid-based modelling.

\section{Grid-based Modelling (GBM)}

There are many teams involved in this project and here we show only a small number of interim results that illustrate progress to date. Within most grid-based models, the scaling laws are used to predict asteroseismic parameters from the mass, radius and $T_{\text {eff }}$ of the model stars. However, it is becoming clear that it is not appropriate to use strict homology on the upper parts of the red-giant branch. Instead, the $\Delta v$ value for any given model star is calculated directly from the computed oscillation frequencies of the model star. For the results presented here, this method has been adopted. In figure 2 , we show representative results from the Silva Aguirre GBM pipeline[12]. The difference 
between the grid mass and the scaling-law mass are plotted as a function of $v_{\max }$ for a variety of different seismic inputs. There is a clear trend in the difference between the two types of mass estimates. The scaling-law approach returns masses significantly higher than the GBM approach, particularly so for low $v_{\max }$ stars. This is due to the correction used within the models for the lack of homology high on the red-giant branch. The different seismic methods are giving similar but not identical results. Even tighter agreement between the different seismic inputs is seen in figure 3 where we show the results from Serenelli[13] extended to include the average seismic parameters. In this case, extra scaling is used to remove the effects of the different solar reference values for the different seismic methods. The level of the difference between the GBM mass and the scaling-law mass at low $v_{\max }$ is consistent with the results in figure 2. For the red-clump stars, the situation is less good. We do not show any results here, but there is disagreement and scatter in the results at the 15 to $20 \%$ level.

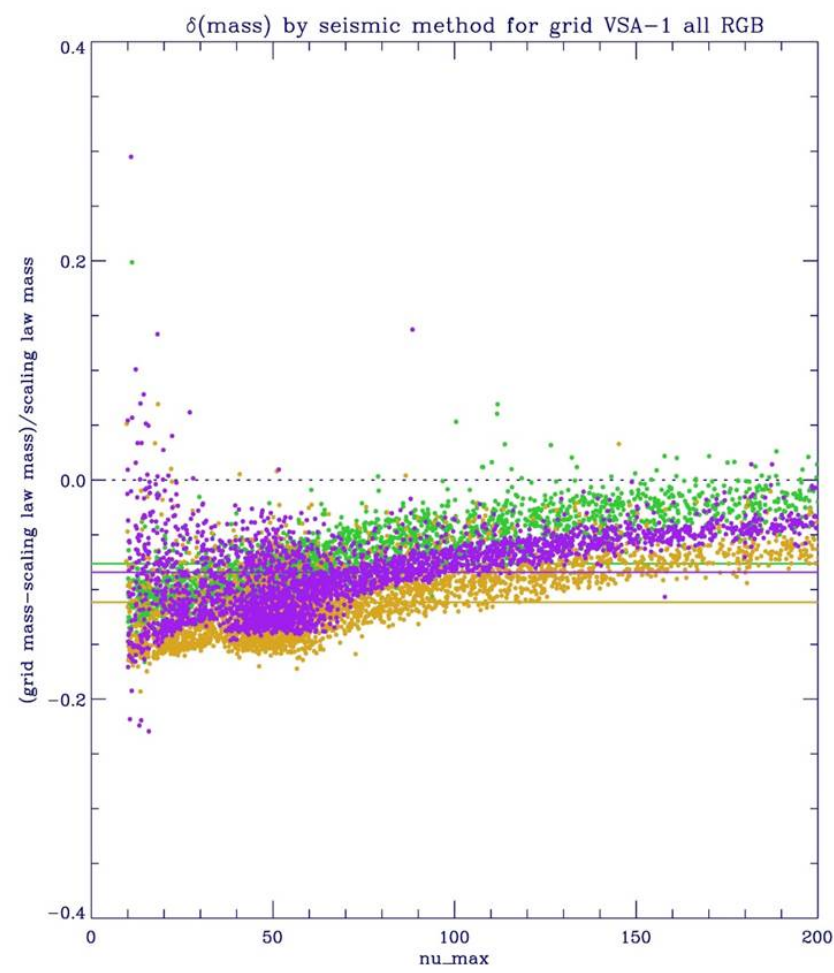

Figure 2: comparison between Mass results from the Silva Aguirre GBM pipeline and scaling-law masses for 3 different seismic input methods - CAN (green), COR(purple) and OCT(light brown)

There is one other very interesting effect that is seen in all the pipelines. The grid-based modelling process selects a model star that best fits the full set of input data. However, the parameters of the chosen model are never identically the same as those of the real, observed star. The input parameters are the average seismic parameters, $v_{\max }$ and $\Delta v$, plus the effective temperature $T_{\text {eff }}$. No particular trends have been seen in the average seismic param-

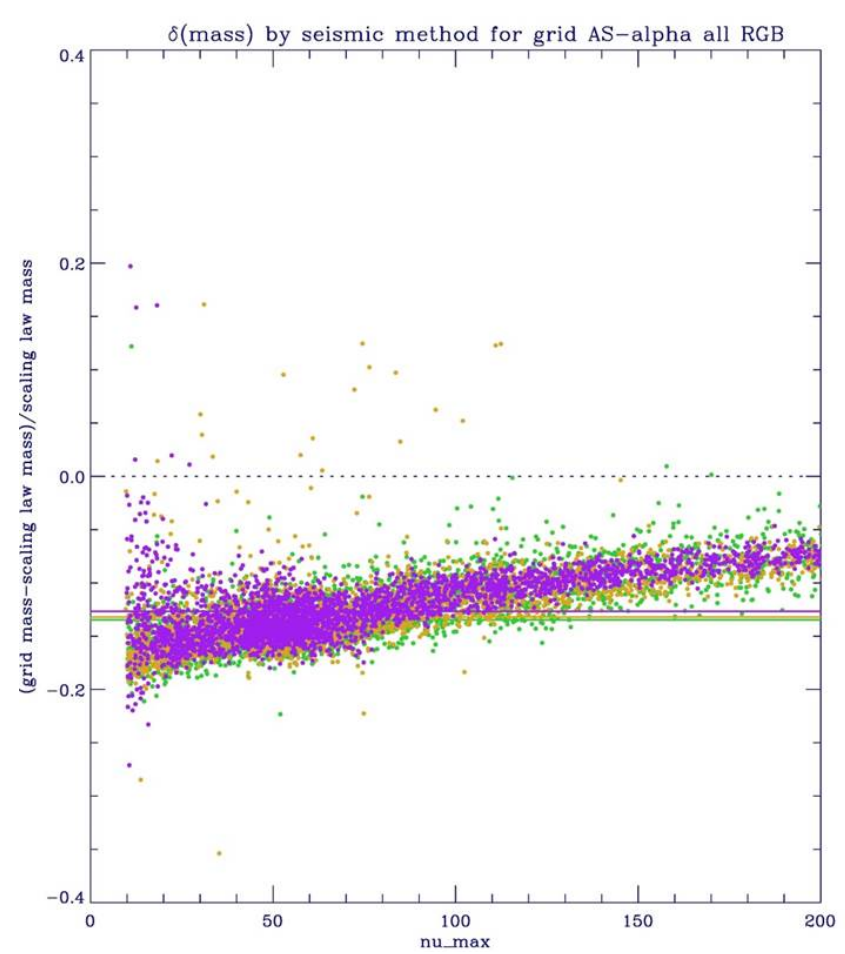

Figure 3: Comparison between Mass results from the Serenelli GBM pipeline and scaling-law masses for 3 different seismic input methods - CAN(green), COR(purple) and OCT(light brown)

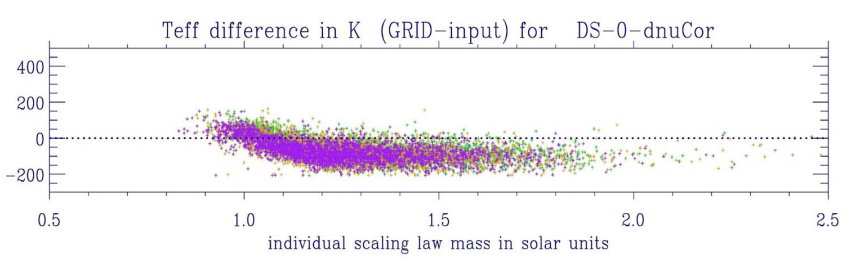

Figure 4: Comparison between the temperature of the chosen model and the observed input temperature from the Stello GBM pipeline for 3 different seismic input methods - CAN(green), COR(purple) and OCT(light brown). The independent variable is the scaling law mass.

eters but there is an effect for the temperature difference between the observed and selected model values. Here we illustrate the effect with results from the Stello GBM pipeline[14] [15] as shown in figure 4 where the temperature difference is plotted as a function of the scaling law mass. At scaling law masses above about $1.2 M_{\odot}$ the chosen model has a lower temperature than the real star by about $100 \mathrm{~K}$. The size of this offset is dependent on the actual GBM pipeline and some have very small offsets. However, as the scaling law mass considered is reduced there is a clear trend in the temperature difference between the real star and the chosen model in the sense that the chosen model temperature gets closer to the observed one and then goes above it. This trend has the effect that the mass of the chosen is increased with respect to the scaling law mass. The GBM pipelines do not return very law mass 
stars. We are currently exploring possible reasons for this effect.

\section{Conclusions}

This is an interim report on the first time that a significant range of average asteroseismic parameters have been used as inputs to grid-based modelling with corrections for the lack of homology for RGB stars. The work is undertaken in the framework of APOKASC. The initial results are very encouraging. One effect at low scaling law mass is curious and needs further investigation.

\section{Acknowledgments}

YE acknowledges the support of the UK Science and Technology Facilities Council (STFC). Funding for the Stellar Astrophysics Centre (SAC) is provided by The Danish National Research Foundation (Grant agreement no.: DNRF106).

\section{References}

[1] M.H. Pinsonneault, Y. Elsworth, C. Epstein, S. Hekker, S. Mészáros, W.J. Chaplin, J.A. Johnson, R.A. García, J. Holtzman, S. Mathur et al., ApJS, 215, 19 (2014)

[2] S. Mathur, R.A. García, C. Régulo, O.L. Creevey, J. Ballot, D. Salabert, T. Arentoft, P.O. Quirion, W.J. Chaplin, H. Kjeldsen, A\&A, 511, A46 (2010)

[3] T. Kallinger, B. Mosser, S. Hekker, D. Huber, D. Stello, S. Mathur, S. Basu, T.R. Bedding, W.J. Chaplin, J. De Ridder et al., A\&A, 522, A1 (2010)

[4] B. Mosser, K. Belkacem, M.J. Goupil, E. Michel, Y. Elsworth, C. Barban, T. Kallinger, S. Hekker, J. De Ridder, R. Samadi et al., A\&A, 525, L9 (2011)
[5] S. Hekker, A.M. Broomhall, W.J. Chaplin, Y.P. Elsworth, S.T. Fletcher, R. New, T. Arentoft, P.O. Quirion, H. Kjeldsen, MNRAS, 402, 2049 (2010)

[6] D. Huber, D. Stello, T.R. Bedding, W.J. Chaplin, T. Arentoft, P.O. Quirion, H. Kjeldsen, Communications in Asteroseismology 160, 74 (2009)

[7] R. Handberg, M.N. Lund, MNRAS, 445, 2698 (2014)

[8] R.A. García, S. Hekker, D. Stello, J. Gutiérrez-Soto, R. Handberg, D. Huber, C. Karoff, K. Uytterhoeven, T. Appourchaux, W.J. Chaplin et al., MNRAS, 414, L6 (2011)

[9] SDSS Collaboration, F.D. Albareti, C. Allende Prieto, A. Almeida, F. Anders, S. Anderson, B.H. Andrews, A. Aragon-Salamanca, M. ArgudoFernandez, E. Armengaud et al., ArXiv e-prints (2016), 1608.02013

[10] H. Kjeldsen, T.R. Bedding, A\&A, 293 (1995)

[11] S. Hekker, Y. Elsworth, B. Mosser, T. Kallinger, W.J. Chaplin, J. De Ridder, R.A. García, D. Stello, B.D. Clarke, J.R. Hall et al., A\&A, 544, A90 (2012)

[12] V. Silva Aguirre, G.R. Davies, S. Basu, J. Christensen-Dalsgaard, O. Creevey, T.S. Metcalfe, T.R. Bedding, L. Casagrande, R. Handberg, M.N. Lund et al., MNRAS, 452, 2127 (2015)

[13] A.M. Serenelli, M. Bergemann, G. Ruchti, L. Casagrande, MNRAS, 429, 3645 (2013)

[14] D. Stello, W.J. Chaplin, H. Bruntt, O.L. Creevey, A. García-Hernández, M.J.P.F.G. Monteiro, A. Moya, P.O. Quirion, S.G. Sousa, J.C. Suárez et al., ApJ, 700, 1589 (2009)

[15] S. Sharma, D. Stello, J. Bland-Hawthorn, D. Huber, T.R. Bedding, ApJ, 822, 15 (2016) 